\title{
Plasma fluctuations as Markovian noise
}

\author{
B. Li, ${ }^{*}$ R. D. Hazeltine, ${ }^{\dagger}$ and K. W. Gentle \\ Department of Physics, Institute for Fusion Studies and Fusion Research Center, \\ The University of Texas at Austin, Austin, Texas 78712, USA
}

(Received 28 August 2007; revised manuscript received 7 November 2007; published 12 December 2007)

\begin{abstract}
Noise theory is used to study the correlations of stationary Markovian fluctuations that are homogeneous and isotropic in space. The relaxation of the fluctuations is modeled by the diffusion equation. The spatial correlations of random fluctuations are modeled by the exponential decay. Based on these models, the temporal correlations of random fluctuations, such as the correlation function and the power spectrum, are calculated. We find that the diffusion process can give rise to the decay of the correlation function and a broad frequency spectrum of random fluctuations. We also find that the transport coefficients may be estimated by the correlation length and the correlation time. The theoretical results are compared with the observed plasma density fluctuations from the tokamak and helimak experiments.
\end{abstract}

DOI: 10.1103/PhysRevE.76.066402

\section{INTRODUCTION}

Fluctuation phenomena are important to many physical systems, such as magnetically confined plasmas [1]. Various methods have been developed for solving fluctuation problems [2-11]. In this paper, we treat random fluctuations as noise and use noise theory $[12,13]$ to study stationary Markovian fluctuations that are statistically homogeneous and isotropic in space. This paper is an extension of the basic formalism outlined in Ref. [14]. We have attempted to clarify the theoretical argument of Ref. [14], by including more details and by various other improvements. In particular the formulas for the fluctuation spectra derived in Ref. [14] assuming time reversibility are shown here to be valid in general. The comparison to experimental data is considerably enlarged, including both more data and a more thorough analysis of the data.

The paper is organized as follows. In Secs. I and II, we review the main conclusions of noise theory [12-14] concerning random fluctuations. In Sec. III, we use the diffusion model to calculate the temporal correlations of random fluctuations. In Sec. IV, we apply the convection-diffusion model to the magnetized plasma fluctuations. In Sec. V, we compare noise theory with the observed plasma density fluctuations during a steady-state discharge.

Let us consider a system described by a set of macroscopic quantities $\boldsymbol{a}(t)=\left\{a_{n}(t)\right\}$, each of which is treated as a stochastic process. The fluctuations are defined as $\boldsymbol{\alpha}(t)$ $=\boldsymbol{a}(t)-\langle\boldsymbol{a}(t)\rangle$, where $\langle\boldsymbol{a}(t)\rangle$ are the equilibrium values of the system, and $\langle\cdots\rangle$ denotes an ensemble average. By definition, the fluctuations have zero mean value $\left\langle\alpha_{n}(t)\right\rangle=0$.

For stationary processes, the probability distributions governing the fluctuations are invariant under translations in time, so that the temporal correlations between two times can only depend on the time differences, $\tau=t-t^{\prime}$. Hence we can write the correlation function as

\footnotetext{
*leehaoq@physics.utexas.edu

${ }^{\dagger}$ rdh@physics.utexas.edu

tk.gentle@mail.utexas.edu
}

PACS number(s): 52.25.Gj, 52.35.Ra, 47.27.Gs, 05.40.-a

$$
C_{n m}(\tau)=\left\langle\alpha_{n}(t) \alpha_{m}\left(t^{\prime}\right)\right\rangle=\left\langle\alpha_{n}(t) \alpha_{m}(t-\tau)\right\rangle .
$$

For stationary processes, the real quantity $\alpha_{n}(t)$ can be expressed as Fourier integrals,

$$
\alpha_{n}(t)=\int_{-\infty}^{\infty} \frac{d \omega}{2 \pi} e^{i \omega t} \alpha_{n}(\omega),
$$

where $\omega=2 \pi f$, and $\alpha_{n}(\omega)$ denotes the Fourier transform of $\alpha_{n}(t)$. The correlation function can then be written as

$$
\left\langle\alpha_{n}(t) \alpha_{m}\left(t^{\prime}\right)\right\rangle=\int \frac{d \omega}{2 \pi} \int \frac{d \omega^{\prime}}{2 \pi} e^{i\left(\omega t-\omega^{\prime} t^{\prime}\right)}\left\langle\alpha_{n}(\omega) \alpha_{m}^{*}\left(\omega^{\prime}\right)\right\rangle,
$$

where the asterisk denotes the complex conjugate.

For stationary processes, $\left\langle\alpha_{n}(t) \alpha_{m}\left(t^{\prime}\right)\right\rangle$ should be a function only of $\tau=t-t^{\prime}$. This immediately tells us that the ensemble average $\left\langle\alpha_{n}(\omega) \alpha_{m}^{*}\left(\omega^{\prime}\right)\right\rangle$ must be proportional to a delta function, $\delta\left(\omega-\omega^{\prime}\right)$. That is, we can write

$$
\left\langle\alpha_{n}(\omega) \alpha_{m}^{*}\left(\omega^{\prime}\right)\right\rangle=S_{n m}(\omega) 2 \pi \delta\left(\omega-\omega^{\prime}\right),
$$

where $S_{n m}(\omega)$ is the power spectral density, also called the power spectrum. Inserting Eq. (4) into Eq. (3), we have

$$
C_{n m}(\tau)=\int_{-\infty}^{\infty} \frac{d \omega}{2 \pi} e^{i \omega \tau} S_{n m}(\omega) .
$$

Thus the power spectrum is equal to the Fourier transform of the correlation function. That is, we can write

$$
S_{n m}(\omega)=\int_{-\infty}^{\infty} d \tau e^{-i \omega \tau} C_{n m}(\tau) .
$$

Equation (5) or (6) state the Wiener-Khintchin theorem. We next write Eq. (6) as

$$
S(\omega)=S_{+}(\omega)+S_{-}(\omega),
$$

defining 


$$
\boldsymbol{S}_{+}(\omega)=\int_{0}^{\infty} d \tau e^{-i \omega \tau} \boldsymbol{C}(\tau)
$$

and

$$
\boldsymbol{S}_{-}(\omega)=\int_{-\infty}^{0} d \tau e^{-i \omega \tau} \boldsymbol{C}(\tau) .
$$

Since $\boldsymbol{C}(\tau)=\boldsymbol{C}^{T}(-\tau)$, it follows from Eqs. (8) and (9) that

$$
S_{-}(\omega)=S_{+}^{T *}(\omega)=S_{+}^{T}(-\omega),
$$

where $T$ denotes the transpose matrix. Thus Eq. (7) can be rewritten as

$$
S(\omega)=S_{+}(\omega)+S_{+}^{* T}(\omega) .
$$

A Markov process is characterized by the conditional probability $P\left(\boldsymbol{\alpha}, t \mid \boldsymbol{\alpha}_{0}\right)$, which measures the probability of observing the value $\boldsymbol{\alpha}$ at time $t>0$, given the initial perturbation $\boldsymbol{\alpha}(t=0)=\boldsymbol{\alpha}_{0}$. The conditional mean is defined by

$$
\langle\boldsymbol{\alpha}(t)\rangle_{0}=\int d \boldsymbol{\alpha} P\left(\boldsymbol{\alpha}, t \mid \boldsymbol{\alpha}_{0}\right) \boldsymbol{\alpha},
$$

where $\langle\cdots\rangle_{0}$ denotes the conditional mean. For Markov processes, the relaxation of the conditional mean can be described by $[12]$

$$
\frac{\partial}{\partial t}\left\langle\alpha_{n}(t)\right\rangle_{0}=-\sum_{m} \Lambda_{n m}\left\langle\alpha_{m}(t)\right\rangle_{0},
$$

where $\Lambda$ is called the response matrix. The formal solution of Eq. (13) is given by

$$
\langle\boldsymbol{\alpha}(t)\rangle_{0}=e^{-\boldsymbol{\Lambda} t} \cdot \boldsymbol{\alpha}_{0}, \text { for } t>0 .
$$

It follows from the Markovian relaxation Eq. (13) that the correlation function takes the form

$$
\boldsymbol{C}(\tau)=e^{-\boldsymbol{\Lambda} \tau} \cdot \boldsymbol{C}(\tau=0), \text { for } \tau>0 .
$$

Inserting Eq. (15) into Eq. (8), we obtain the formal expression

$$
S_{+}(\omega)=(\Lambda+i \omega I)^{-1} \cdot C(\tau=0)
$$

or

$$
(\Lambda+i \omega I) \cdot S_{+}(\omega)=C(\tau=0),
$$

where $I$ is the unit matrix. Thus the power spectrum Eq. (11) is known in terms of the response matrix $\Lambda$ and the singletime correlations $\boldsymbol{C}(\tau=0)$.

\section{SPACE-TIME CORRELATIONS}

In the turbulent state, the system is irregular in both space and time. Therefore we need to study both spatial and temporal correlations of random fluctuations. For homogeneous statistics, the probability distributions governing the fluctuations are invariant under translations in space, so that the spatial correlations between two points can only depend on the spatial coordinate differences, $\boldsymbol{r}=\boldsymbol{x}-\boldsymbol{x}^{\prime}$.
In this paper, we consider the fluctuations in a single quantity at different spatial points, $\alpha(\boldsymbol{x}, t)$. Thus the discrete index $n$ in the formalism corresponds to the spatial coordinate $\boldsymbol{x}$. For instance, the fluctuations $\alpha_{n}(t) \rightarrow \alpha(\boldsymbol{x}, t)$.

Another change in the formalism is that for homogeneous statistics, the matrix products become convolution integrals. For instance, the relaxation of the conditional mean Eq. (13) becomes

$$
\frac{\partial}{\partial t}\langle\alpha(\boldsymbol{x}, t)\rangle_{0}+\int d^{3} x^{\prime} \Lambda\left(\boldsymbol{x}-\boldsymbol{x}^{\prime}\right)\left\langle\alpha\left(\boldsymbol{x}^{\prime}, t\right)\right\rangle_{0}=0 .
$$

Let us consider stationary and homogeneous statistics. The definition of the correlation function Eq. (1) then becomes

$$
C(\boldsymbol{r}, \tau)=\left\langle\alpha(\boldsymbol{x}, t) \alpha\left(\boldsymbol{x}^{\prime}, t^{\prime}\right)\right\rangle .
$$

Similarly, the definition of the power spectrum Eq. (4) becomes

$$
\left\langle\alpha(\boldsymbol{x}, \omega) \alpha^{*}\left(\boldsymbol{x}^{\prime}, \omega^{\prime}\right)\right\rangle=S(\boldsymbol{r}, \omega) 2 \pi \delta\left(\omega-\omega^{\prime}\right),
$$

where $\alpha(\boldsymbol{x}, \omega)$ denotes the temporal Fourier transform of $\alpha(\boldsymbol{x}, t)$. In view of Eqs. (5) and (6), we have the WienerKhintchin theorem:

$$
C(\boldsymbol{r}, \tau)=\int_{-\infty}^{\infty} \frac{d \omega}{2 \pi} e^{i \omega \tau} S(\boldsymbol{r}, \omega)
$$

and

$$
S(\boldsymbol{r}, \omega)=\int_{-\infty}^{\infty} d \tau e^{-i \omega \tau} C(\boldsymbol{r}, \tau) .
$$

Note that the variance, i.e., the mean square value of the fluctuations, is given by

$$
C(\boldsymbol{r}=0, \quad \tau=0)=\left\langle\alpha^{2}\right\rangle=\sigma^{2},
$$

where $\sigma$ is the standard deviation. It follows from Eq. (21) that the fluctuation energy is given by

$$
\left\langle\alpha^{2}\right\rangle=\int_{-\infty}^{\infty} \frac{d \omega}{2 \pi} S(\boldsymbol{r}=0, \omega)=\int_{-\infty}^{\infty} d f S(f) .
$$

It is clear that the autopower spectral density $S(\boldsymbol{r}=0, \omega)$ indicates the frequency distribution of the fluctuation energy, and $S(f) d f$ specifies the fluctuation power in the frequency interval $d f$.

For homogeneous statistics, the fluctuating quantity $\alpha(\boldsymbol{x}, t)$ may be written as Fourier integrals,

$$
\alpha(\boldsymbol{x}, t)=\int \frac{d^{3} k}{(2 \pi)^{3}} e^{i k \cdot x} \alpha(\boldsymbol{k}, t),
$$

where $\boldsymbol{k}$ is the wave vector, and $\alpha(\boldsymbol{k}, t)$ denotes the spatial Fourier transform of $\alpha(\boldsymbol{x}, t)$. The statistical stationarity and homogeneity of the fluctuations require that $\left\langle\alpha(\boldsymbol{x}, t) \alpha\left(\boldsymbol{x}^{\prime}, t^{\prime}\right)\right\rangle$ be a function only of $\boldsymbol{r}=\boldsymbol{x}-\boldsymbol{x}^{\prime}$ and $\tau=t-t^{\prime}$. This immediately tells us that the ensemble average $\left\langle\alpha(\boldsymbol{k}, t) \alpha^{*}\left(\boldsymbol{k}^{\prime}, t^{\prime}\right)\right\rangle$ must be proportional to a delta function, $\delta^{3}\left(\boldsymbol{k}-\boldsymbol{k}^{\prime}\right)$. That is, we can write 


$$
\left\langle\alpha(\boldsymbol{k}, t) \alpha^{*}\left(\boldsymbol{k}^{\prime}, t^{\prime}\right)\right\rangle=C(\boldsymbol{k}, \tau)(2 \pi)^{3} \delta^{3}\left(\boldsymbol{k}-\boldsymbol{k}^{\prime}\right),
$$

where $C(\boldsymbol{k}, \tau)$ is called the wave-number spectrum. Note that $C(\boldsymbol{k}, \tau=0)$ is real, by definition. The correlation function can then be expressed as

$$
C(\boldsymbol{r}, \tau)=\int \frac{d^{3} k}{(2 \pi)^{3}} e^{i \boldsymbol{k} \cdot \boldsymbol{r}} C(\boldsymbol{k}, \tau) .
$$

Thus $C(\boldsymbol{k}, \tau)$ is equal to the spatial Fourier transform of the correlation function.

Similarly, if we let $\alpha(\boldsymbol{k}, \omega)$ denote the space-time Fourier transform of $\alpha(\boldsymbol{x}, t)$, then the ensemble average $\left\langle\alpha(\boldsymbol{k}, \omega) \alpha^{*}\left(\boldsymbol{k}^{\prime}, \omega^{\prime}\right)\right\rangle$ must be proportional to the delta functions, $\delta^{3}\left(\boldsymbol{k}-\boldsymbol{k}^{\prime}\right) \delta\left(\omega-\omega^{\prime}\right)$. That is, we can write

$$
\left\langle\alpha(\boldsymbol{k}, \omega) \alpha^{*}\left(\boldsymbol{k}^{\prime}, \omega^{\prime}\right)\right\rangle=S(\boldsymbol{k}, \omega)(2 \pi)^{4} \delta^{3}\left(\boldsymbol{k}-\boldsymbol{k}^{\prime}\right) \delta\left(\omega-\omega^{\prime}\right),
$$

where $S(\boldsymbol{k}, \omega)$ is called the wave-number-frequency spectrum. Note that $S(\boldsymbol{k}, \omega)$ is real, by definition. The correlation function can then be written as

$$
C(\boldsymbol{r}, \tau)=\int_{-\infty}^{\infty} \frac{d \omega}{2 \pi} e^{i \omega \tau} \int \frac{d^{3} k}{(2 \pi)^{3}} e^{i \boldsymbol{k} \cdot \boldsymbol{r}} S(\boldsymbol{k}, \omega) .
$$

Thus $S(\boldsymbol{k}, \omega)$ is equal to the space-time Fourier transforms of the correlation function.

Comparing Eq. (21) with Eq. (29), we see that

$$
S(\boldsymbol{r}, \omega)=\int \frac{d^{3} k}{(2 \pi)^{3}} e^{i \boldsymbol{k} \cdot \boldsymbol{r}} S(\boldsymbol{k}, \omega) .
$$

Thus $S(\boldsymbol{k}, \omega)$ is equal to the spatial Fourier transform of the power spectrum. In particular, the autopower spectrum is given by

$$
S(\boldsymbol{r}=0, \omega)=\int \frac{d^{3} k}{(2 \pi)^{3}} S(\boldsymbol{k}, \omega) .
$$

Comparing Eq. (27) with Eq. (29), we see that

$$
C(\boldsymbol{k}, \tau)=\int_{-\infty}^{\infty} \frac{d \omega}{2 \pi} e^{i \omega \tau} S(\boldsymbol{k}, \omega) .
$$

Thus $S(\boldsymbol{k}, \omega)$ is equal to the temporal Fourier transform of $C(\boldsymbol{k}, \tau)$. Note that Eq. (32) is the spatial Fourier transform of the Wiener-Khintchin theorem Eq. (21). It follows from Eq. (32) that $S(\boldsymbol{k}, \omega)$ must satisfy the condition

$$
C(\boldsymbol{k}, \tau=0)=\int_{-\infty}^{\infty} \frac{d \omega}{2 \pi} S(\boldsymbol{k}, \omega) .
$$

In view of Eq. (7), we next write Eq. (22) as

$$
S(\boldsymbol{r}, \omega)=S_{+}(\boldsymbol{r}, \omega)+S_{-}(\boldsymbol{r}, \omega),
$$

defining

$$
S_{+}(\boldsymbol{r}, \omega)=\int_{0}^{\infty} d \tau e^{-i \omega \tau} C(\boldsymbol{r}, \tau),
$$

and

$$
S_{-}(\boldsymbol{r}, \omega)=\int_{-\infty}^{0} d \tau e^{-i \omega \tau} C(\boldsymbol{r}, \tau) .
$$

The spatial Fourier transform of Eq. (34) gives

$$
S(\boldsymbol{k}, \omega)=S_{+}(\boldsymbol{k}, \omega)+S_{-}(\boldsymbol{k}, \omega),
$$

where $S_{+}(k, \omega)$ and $S_{-}(k, \omega)$ denote the spatial Fourier transforms of $S_{+}(r, \omega)$ and $S_{-}(r, \omega)$, respectively. It follows from Eq. (35) that

$$
S_{+}(\boldsymbol{k}, \omega)=\int d^{3} r e^{-i \boldsymbol{k} \cdot \boldsymbol{r}} \int_{0}^{\infty} d \tau e^{-i \omega \tau} C(\boldsymbol{r}, \tau) .
$$

Note that the transpose matrix corresponds to the transpose on $\boldsymbol{x}$ and $\boldsymbol{x}^{\prime}$. For instance, $\boldsymbol{S}^{T}(\omega) \rightarrow S_{m n}(\omega) \rightarrow S\left(\boldsymbol{x}^{\prime}, \boldsymbol{x}, \omega\right)$ $=S(-\boldsymbol{r}, \omega)$. Thus for homogeneous statistics, Eq. (10) implies that

$$
S_{-}(\boldsymbol{r}, \omega)=S_{+}(-\boldsymbol{r},-\omega) .
$$

The spatial Fourier transform of Eq. (39) then gives

$$
S_{-}(\boldsymbol{k}, \omega)=S_{+}(-\boldsymbol{k},-\omega)=S_{+}^{*}(\boldsymbol{k}, \omega) .
$$

Thus Eq. (37) can be rewritten as

$$
S(\boldsymbol{k}, \omega)=2 \operatorname{Re} S_{+}(\boldsymbol{k}, \omega),
$$

where Re indicates taking the real part.

Finally, we derive the fluctuation spectra based on the Markovian relaxation Eq. (18). For homogeneous statistics, Eq. (17) becomes

$$
\begin{aligned}
& \int d^{3} x^{\prime \prime}\left[\Lambda\left(\boldsymbol{x}-\boldsymbol{x}^{\prime \prime}\right)+i \omega \delta\left(\boldsymbol{x}-\boldsymbol{x}^{\prime \prime}\right)\right] S_{+}\left(\boldsymbol{x}^{\prime \prime}-\boldsymbol{x}^{\prime}, \omega\right) \\
& =C\left(\boldsymbol{x}-\boldsymbol{x}^{\prime}, \quad \tau=0\right) .
\end{aligned}
$$

The spatial Fourier transform of the convolution integral Eq. (42) then gives

$$
S_{+}(\boldsymbol{k}, \omega)=\frac{C(\boldsymbol{k}, \tau=0)}{\Lambda(\boldsymbol{k})+i \omega},
$$

where $\Lambda(\boldsymbol{k})$ denotes the spatial Fourier transform of $\Lambda(\boldsymbol{r})$. Inserting Eq. (43) into Eq. (41), we obtain the wave-numberfrequency spectrum

$$
S(\boldsymbol{k}, \omega)=2 \operatorname{Re}\left[\frac{C(\boldsymbol{k}, \tau=0)}{\Lambda(\boldsymbol{k})+i \omega}\right] .
$$

Thus $S(\boldsymbol{k}, \omega)$ is known in terms of $\Lambda(\boldsymbol{k})$ and $C(\boldsymbol{k}, \tau=0)$. In general, $\Lambda(\boldsymbol{k})$ is complex. If we write $\Lambda(\boldsymbol{k})=\Lambda_{r}(\boldsymbol{k})-i \Lambda_{i}(\boldsymbol{k})$, then Eq. (44) becomes

$$
S(\boldsymbol{k}, \omega)=C(\boldsymbol{k}, \tau=0) \frac{2 \Lambda_{r}(\boldsymbol{k})}{\Lambda_{r}^{2}(\boldsymbol{k})+\left[\omega-\Lambda_{i}(\boldsymbol{k})\right]^{2}} .
$$

Thus the frequency dependence of $S(\boldsymbol{k}, \omega)$ takes the Lorentzian form. Equation (45) shows that the imaginary part of $\Lambda(\boldsymbol{k})$ shifts the maximum of the spectra away from zero frequency. 


\section{DIFFUSION MODEL}

For physical systems with diffusion response to random fluctuations, the relaxation of the conditional mean is modeled by the diffusion equation [12]

$$
\frac{\partial}{\partial t}\langle\alpha(\boldsymbol{x}, t)\rangle_{0}=D \nabla^{2}\langle\alpha(\boldsymbol{x}, t)\rangle_{0}
$$

where $D$ is the effective diffusion coefficient. Comparing Eq. (46) with Eq. (18), we obtain the diffusion response function

$$
\Lambda(\boldsymbol{r})=-D \nabla^{2} \delta(\boldsymbol{r}) \text {. }
$$

The spatial Fourier transform of Eq. (47) then gives

$$
\Lambda(k)=D k^{2},
$$

where $k=|\boldsymbol{k}|$ is the magnitude of the wave vector.

For isotropic statistics, the probability distributions governing the fluctuations are invariant under rotations in space, so that the spatial correlations between two points can only depend on the separation distance, $r=|\boldsymbol{r}|$. As a result, the spatial Fourier transforms of the correlations can only depend on the magnitude of the wave vector, $k=|\boldsymbol{k}|$.

Let us consider homogeneous and isotropic statistics. Using Eq. (48) we can write the wave-number-frequency spectrum Eq. (44) as

$$
S(k, \omega)=C(k, \tau=0) \frac{2 D k^{2}}{\left(D k^{2}\right)^{2}+\omega^{2}} .
$$

The inverse temporal Fourier transform of Eq. (49) then gives the wave-number spectrum

$$
C(k, \tau)=C(k, \tau=0) e^{-k^{2} D|\tau|} .
$$

Thus the wave-number dependence of $C(k, \tau)$ takes the Gaussian form. Inserting Eq. (49) into Eq. (31), we obtain the autopower spectrum

$$
S(r=0, \omega)=\int \frac{d^{3} k}{(2 \pi)^{3}} C(k, \tau=0) \frac{2 D k^{2}}{D^{2} k^{4}+\omega^{2}} .
$$

The theory and experiment show that the spatial correlations of fluctuations can be approximated by an exponential decay [15-18]. Hence we assume that the spatial correlations of isotropic fluctuations take the form

$$
\left\langle\alpha(\boldsymbol{x}, t) \alpha\left(\boldsymbol{x}^{\prime}, t\right)\right\rangle=C(\boldsymbol{r}, \tau=0)=\left\langle\alpha^{2}\right\rangle e^{-|\boldsymbol{r}| / \lambda_{c}},
$$

where $\lambda_{c}$ is the correlation length. Note that the slope of the exponential decay is discontinuous at $\boldsymbol{r}=0$. The slope discontinuity reflects inability of the exponential decay to resolve fine structure at the origin [15].

For isotropic statistics, the mean square value of the fluctuations can be written as

$$
\left\langle\alpha^{2}\right\rangle=\int \frac{d^{3} k}{(2 \pi)^{3}} C(k, \tau=0)=\int_{0}^{\infty} d k E(k),
$$

where $E(k)$ is called the energy spectrum. It is clear that the energy spectrum indicates the distribution of the fluctuation energy over different length scales, and $E(k) d k$ specifies the fluctuation power in the wave-number interval $d k$.

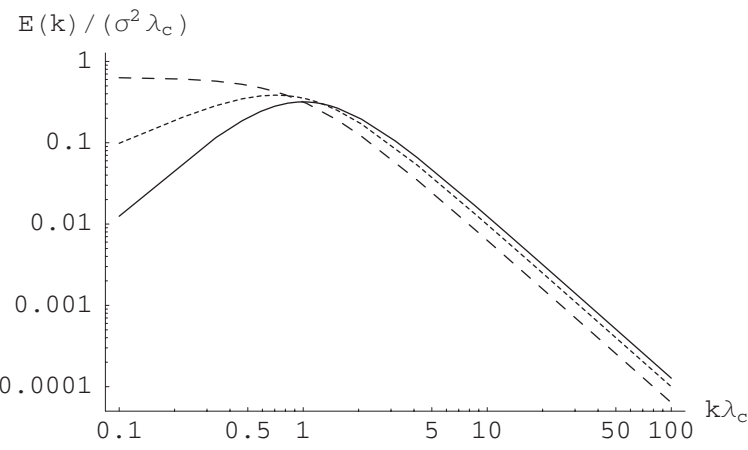

FIG. 1. Comparison of 1D energy spectrum (dashed line), 2D energy spectrum (dotted line), and 3D energy spectrum (solid line). The energy spectrum $E(k)$ is normalized by the variance $\sigma^{2}$ and the correlation length $\lambda_{c}$.

It follows from Eq. (53) that the spatial Fourier transforms of Eq. (52) give the energy spectra of the exponential decay [19]. In three dimensions, the energy spectrum is given by

$$
E(k)=\frac{4 \pi k^{2}}{(2 \pi)^{3}} C(k, \tau=0)=\left\langle\alpha^{2}\right\rangle \frac{4}{\pi} \frac{k^{2} \lambda_{c}^{3}}{\left(1+k^{2} \lambda_{c}^{2}\right)^{2}} .
$$

In two dimensions, the energy spectrum is given by

$$
E(k)=\frac{2 \pi k}{(2 \pi)^{2}} C(k, \tau=0)=\left\langle\alpha^{2}\right\rangle \frac{k \lambda_{c}^{2}}{\left(1+k^{2} \lambda_{c}^{2}\right)^{3 / 2}} .
$$

In one dimension, the energy spectrum is given by

$$
E(k)=\frac{2}{2 \pi} C(k, \tau=0)=\left\langle\alpha^{2}\right\rangle \frac{2}{\pi} \frac{\lambda_{c}}{1+k^{2} \lambda_{c}^{2}} .
$$

As shown in Fig. 1, the energy spectra of the exponential decay have the following key features. At small wave numbers $k \lambda_{c}<1$, the one-dimensional (1D) energy spectrum Eq. (56) becomes flat, while the 3D energy spectrum Eq. (54) drops to zero. At large wave numbers $k \lambda_{c}>1$, the energy spectra become parallel to each other, and the 1D energy spectrum is on the left side of the $3 \mathrm{D}$ energy spectrum. The 2D energy spectrum Eq. (55) is in between of the 1D and 3D energy spectra. The wave-number dependence of $E(k)$ at large wave numbers can be obtained from Eqs. (54)-(56). We find that for $k \lambda_{c}>2 \pi$, regardless of spatial dimensions, the energy spectra of the exponential decay are proportional to the inverse square of wave number:

$$
E(k) \propto k^{-2} .
$$

Since the fluctuation wavelengths of interest are not expected to be infinite, we may introduce a cutoff wave number for the lower limit of the $k$ integrals. On the other hand, for inhomogeneous systems that have gradients in the equilibrium profiles, we exclude very long wavelengths, so that the resulting small-scale fluctuations do not see the variation of the background profiles. The exact cutoff should be given by the diagnostics of the experiments.

The correlation length is often shorter than the length scales of the equilibrium profile gradients. Hence the cutoff wave number is approximated by the minimum wave number of the inertial range: 


$$
k_{c}=\frac{2 \pi}{\lambda_{c}} .
$$

Since the longest wavelength of the inertial range corresponds to the length scale at which the fluctuation energy is introduced, our approximations imply that the mean square value of small-scale fluctuations is determined by the wavelengths that are shorter than the correlation length. Hence the fluctuation energy Eq. (53) is approximated by

$$
\left\langle\alpha^{2}\right\rangle=\int \frac{d^{3} k}{(2 \pi)^{3}} C(k, \tau=0) \approx \int_{k_{c}}^{\infty} d k E(k) .
$$

It follows that the autopower spectrum Eq. (51) becomes

$$
S(r=0, \omega)=\int_{k_{c}}^{\infty} d k E(k) \frac{2 D k^{2}}{D^{2} k^{4}+\omega^{2}},
$$

so that the condition Eq. (24) is satisfied.

The proportionality constant of Eq. (57) is then obtained from the condition Eq. (59). Thus for $k>k_{c}$, regardless of spatial dimensions, we have

$$
E(k)=\left\langle\alpha^{2}\right\rangle \frac{k_{c}}{k^{2}}
$$

Inserting Eq. (61) into Eq. (60), we obtain the autopower spectrum

$$
S(r=0, \omega)=\left\langle\alpha^{2}\right\rangle \int_{k_{c}}^{\infty} d k \frac{2 k_{c} D}{D^{2} k^{4}+\omega^{2}} .
$$

Equation (62) shows that many large wave numbers, i.e., short wavelengths, contribute to the fluctuation power $S(f) d f$ in the frequency interval $d f$.

The diffusion coefficient may be written in the random walk form with the correlation length $\lambda_{c}$ as the step size of the random walk:

$$
D=\frac{\omega_{d}}{k_{c}^{2}}=\frac{f_{d} \lambda_{c}^{2}}{2 \pi},
$$

where the step frequency of the random walk, $\omega_{d}=2 \pi f_{d}$, is called the diffusion frequency.

If we normalize the frequency and wave number according to $\nu=\omega / \omega_{d}=f / f_{d}$ and $q=k / k_{c}$, then $D k^{2}=q^{2} \omega_{d}$ and Eq. (62) becomes

$$
S(\nu) /\left\langle\alpha^{2}\right\rangle=\frac{2}{\omega_{d}} \int_{1}^{\infty} \frac{d q}{q^{4}+\nu^{2}}=\frac{2}{3 \omega_{d}} H\left(-\nu^{2}\right)
$$

or

$$
S(r=0, \omega)=\frac{2\left\langle\alpha^{2}\right\rangle}{3 \omega_{d}} H\left(-\omega^{2} / \omega_{d}^{2}\right),
$$

where $H\left(-\nu^{2}\right)$ represents the hypergeometric function

$$
{ }_{2} F_{1}\left(\frac{3}{4}, 1 ; \frac{7}{4} ;-\nu^{2}\right)=1-\frac{3}{7} \nu^{2}+\frac{3}{11} \nu^{4} \cdots .
$$

Equation (65) is called the diffusion spectrum and it has a finite maximum at zero frequency: $S_{\max }=S(\omega=0)$

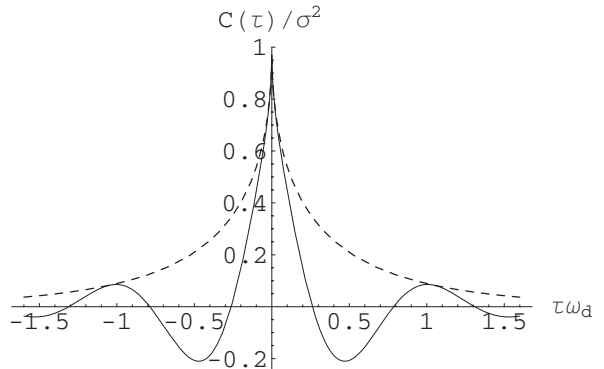

FIG. 2. Comparison of the diffusion decay Eq. (67) (dashed line) and the oscillating decay Eq. (70) with $Q=6$ (solid line). The autocorrelation function $C(\tau)$ is normalized by the variance $\sigma^{2}$.

$=2\left\langle\alpha^{2}\right\rangle /\left(3 \omega_{d}\right)$. For $|\nu|>2, H\left(-\nu^{2}\right) \approx|\nu|^{-1.1}$. That is, in the positive frequency range $f>2 f_{d}$, the diffusion spectrum $S(f) \propto f^{-1.1}$. Thus we obtain the well-known $1 / f$ noise frequency spectrum [5].

The inverse Fourier transform of the autopower spectrum Eq. (65) then gives the autocorrelation function

$$
C(r=0, \tau) /\left\langle\alpha^{2}\right\rangle=e^{-|\tau| \omega_{d}}-\sqrt{\pi|\tau| \omega_{d}} \operatorname{erfc}\left(\sqrt{|\tau| \omega_{d}}\right),
$$

where the complementary error function, $\operatorname{erfc}(z)=1-\operatorname{erf}(z)$, and $\operatorname{erf}(z)$ is the error function. Equation (67) is called the diffusion decay. Note that the slope of the diffusion decay at $\tau=0$ is infinite and discontinuous. The slope discontinuity reflects inability of the Markovian relaxation to resolve fine structure at the origin [12].

The correlation time of the diffusion decay Eq. (67) is given by

$$
\tau_{c}=\int_{0}^{\infty} d \tau C(r=0, \tau) /\left\langle\alpha^{2}\right\rangle=\frac{1}{3 \omega_{d}}=\frac{1}{6 \pi f_{d}} .
$$

The decay of the autocorrelation function Eq. (67) can be characterized by the correlation time, where $C\left(\tau_{c}\right) \approx 0.3\left\langle\alpha^{2}\right\rangle$. Since the power spectrum is the Fourier transform of the correlation function, the decay rate of the autocorrelation function, i.e., the reciprocal of the correlation time, indicates the width of the autopower spectrum $\Delta \omega=1 / \tau_{c}=3 \omega_{d}$ at the height $S(\Delta \omega) \approx 0.3 S_{\max }$. Thus the diffusion frequency $\omega_{d}$ is a measure of the decay rate, i.e., the spectrum width.

Using Eq. (68) we can rewrite the transport coefficient Eq. (63) as

$$
D=\frac{f_{d} \lambda_{c}^{2}}{2 \pi}=\frac{\lambda_{c}^{2}}{12 \pi^{2} \tau_{c}}
$$

Equation (69) shows that the observations of longer correlation lengths or shorter correlation times imply larger transport coefficients. Thus the fluctuation and dissipation are related to each other through the random walk formula.

The experiments observe that often the correlation functions not only decay but also oscillate at a nearly constant frequency [18]. Note that the oscillations are not driven externally, since the amplitude of oscillations decays. The regular oscillations suggest that there is a dominant frequency in the temporal correlations. Hence the oscillating decay of the 


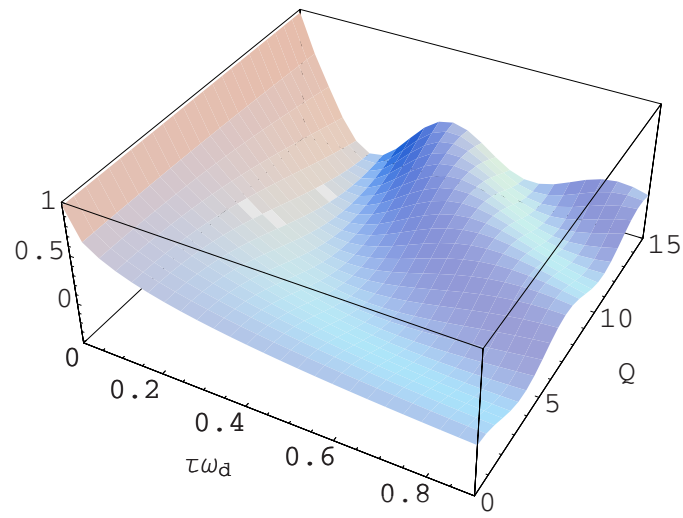

FIG. 3. (Color online) The surface plot of normalized autocorrelation function $C(\tau) / \sigma^{2}$ as a function of normalized time differences $\tau \omega_{d}$ and the quality factor $Q$.

autocorrelation function is simply modeled by a cosine function times the diffusion decay Eq. (67). That is, we write the autocorrelation function as

$$
C(\tau)=\left\langle\alpha^{2}\right\rangle \cos \left(\omega_{0} \tau\right)\left[e^{-|\tau| \omega_{d}}-\sqrt{\pi|\tau| \omega_{d}} \operatorname{erfc}\left(\sqrt{|\tau| \omega_{d}}\right)\right],
$$

where $\omega_{0}=2 \pi f_{0}$ is a constant, and $f_{0}$ is the oscillation frequency. The ratio of the oscillation frequency $f_{0}$ to the decay rate $f_{d}$ may be defined as the quality factor of fluctuations

$$
Q=\frac{\omega_{0}}{\omega_{d}}=\frac{f_{0}}{f_{d}} .
$$

As shown in Figs. 2 and 3, the decay of the autocorrelation function becomes oscillatory for nonzero $Q$ value, and the diffusion decay Eq. (67) is the case for Eq. (70) with $Q=0$.

The Fourier transform of the autocorrelation function Eq. (70) then gives the autopower spectrum

$$
2 S(\omega)=\frac{2\left\langle\alpha^{2}\right\rangle}{3 \omega_{d}}\left\{H\left[-\left(\omega+\omega_{0}\right)^{2} / \omega_{d}^{2}\right]+H\left[-\left(\omega-\omega_{0}\right)^{2} / \omega_{d}^{2}\right]\right\} .
$$

Thus $\omega_{0}$ is also the peak frequency of the spectrum. As shown in Figs. 4 and 5, the peak of the spectra is shifted away from zero frequency for nonzero $Q$ value, and the diffusion spectrum Eq. (65) is the case for Eq. (72) with $Q=0$.

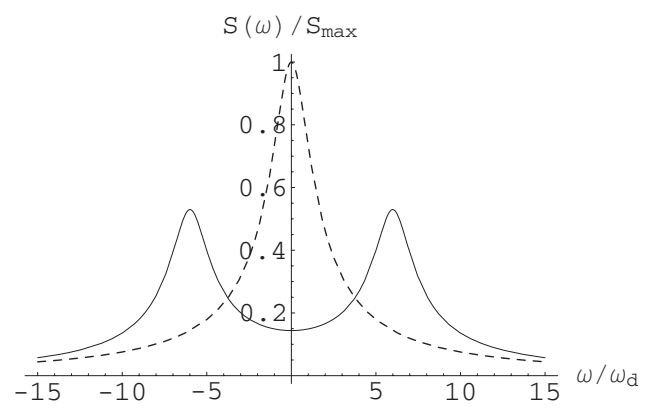

FIG. 4. Comparison of the diffusion spectrum Eq. (65) (dashed line) and the shifted spectrum Eq. (72) with $Q=6$ (solid line). The autopower spectrum $S(\omega)$ is normalized by $S_{\max }=2\left\langle\alpha^{2}\right\rangle /\left(3 \omega_{d}\right)$.

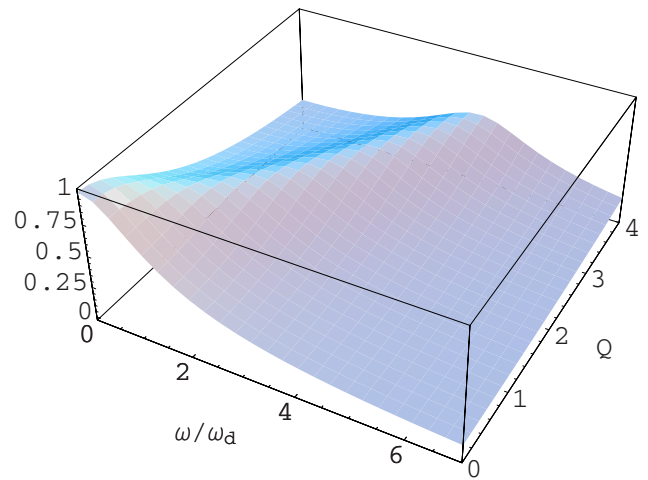

FIG. 5. (Color online) The surface plot of normalized autopower spectrum $S(\omega) / S_{\max }$ as a function of normalized frequency $\omega / \omega_{d}$ and the quality factor $Q$.

Since the autopower spectrum is even in $\omega$, it follows from Eq. (24) that the fluctuation power can be expressed as

$$
\left\langle\alpha^{2}\right\rangle=2 \int_{0}^{\infty} d f S(f)=\int_{0}^{\infty} d f G(f),
$$

where $G(f)=2 S(f)$ is the frequency spectrum usually measured in the experiments. Note that the spectral density $G(f)$ is defined to only have positive frequencies. It is clear that the autopower spectrum has units of power per frequency, here the power refers to the units of $\left\langle\alpha^{2}\right\rangle$. Using Eq. (72) we obtain the frequency spectrum

$$
G(f)=\frac{\left\langle\alpha^{2}\right\rangle}{3 \pi f_{d}}\left\{H\left[-\left(f+f_{0}\right)^{2} / f_{d}^{2}\right]+H\left[-\left(f-f_{0}\right)^{2} / f_{d}^{2}\right]\right\} .
$$

Note that the cutoff wavelength, i.e., the correlation length, $\lambda_{c}$ does not enter the final results of the autocorrelations.

\section{CONVECTION-DIFFUSION MODEL}

For a wide variety of physical situations, the relaxation of fluctuations involves a combination of fluid motion and diffusion. Hence the evolution of the conditional mean is modeled by the convection-diffusion equation [12],

$$
\frac{\partial}{\partial t}\langle\alpha(\boldsymbol{x}, t)\rangle_{0}+\boldsymbol{U} \cdot \boldsymbol{\nabla}\langle\alpha(\boldsymbol{x}, t)\rangle_{0}=D \boldsymbol{\nabla}^{2}\langle\alpha(\boldsymbol{x}, t)\rangle_{0},
$$

where $\boldsymbol{U}$ is the effective flow velocity.

Comparing Eq. (75) with Eq. (18), we obtain the convection-diffusion response function

$$
\Lambda(\boldsymbol{r})=-D \boldsymbol{\nabla}^{2} \delta(\boldsymbol{r})-\boldsymbol{U} \cdot \boldsymbol{\nabla} \delta(\boldsymbol{r}) .
$$

The Fourier transform of Eq. (76) then gives

$$
\Lambda(\boldsymbol{k})=D k^{2}-i \boldsymbol{k} \cdot \boldsymbol{U} .
$$

Note that in the convection-diffusion model $\Lambda(\boldsymbol{k})$ is complex and anisotropy enters through its imaginary part. Inserting Eq. (77) into Eq. (44), we obtain the wave-number-frequency spectrum 


$$
S(\boldsymbol{k}, \omega)=C(\boldsymbol{k}, \tau=0) \frac{2 D k^{2}}{D^{2} k^{4}+(\omega-\boldsymbol{k} \cdot \boldsymbol{U})^{2}} .
$$

Note that the Lorentzian form of $S(\boldsymbol{k}, \omega)$ satisfies the condition Eq. (33).

We next apply the convection-diffusion model to the fluctuations in the magnetized plasma. Because of the strong magnetic fields, the relaxation of the fluctuations is anisotropic in the magnetized plasma. We expect that fluid motion is the dominant transport along the magnetic field, while diffusion is the dominant transport in the two dimensions perpendicular to the magnetic field. Hence we write Eq. (75) as

$$
\frac{\partial}{\partial t}\langle\alpha(\boldsymbol{x}, t)\rangle_{0}+U_{\|} \nabla_{\|}\langle\alpha(\boldsymbol{x}, t)\rangle_{0}=D_{\perp} \nabla_{\perp}^{2}\langle\alpha(\boldsymbol{x}, t)\rangle_{0},
$$

where $U_{\|}$is the effective flow velocity along the magnetic field, and $D_{\perp}$ represents the effective diffusion perpendicular to the magnetic field. Then Eq. (77) becomes

$$
\Lambda(\boldsymbol{k})=D_{\perp} k_{\perp}^{2}-i k_{\|} U_{\|} \cdot
$$

Consequently the wave-number-frequency spectrum Eq. (78) becomes

$$
S(\boldsymbol{k}, \omega)=C(\boldsymbol{k}, \tau=0) \frac{2 D_{\perp} k_{\perp}^{2}}{D_{\perp}^{2} k_{\perp}^{4}+\left(\omega-k_{\|} U_{\|}\right)^{2}} .
$$

In the magnetized plasma, the parallel correlation length $\lambda_{c \|}$ is much longer than the perpendicular correlation length $\lambda_{c \perp}$. Hence we assume that the spatial correlations of the fluctuations take the form

$$
C(\boldsymbol{r}, \tau=0)=\left\langle\alpha^{2}\right\rangle e^{-\left|\boldsymbol{r}_{\perp}\right| / \lambda_{c \perp}} \cos \left(k_{0} r_{\|}\right),
$$

where the constant $k_{0}$ indicates a dominant parallel wave number. It follows from Eqs. (81) and (82) that the autopower spectrum Eq. (31) becomes

$$
\begin{aligned}
S(\omega)= & \int_{-\infty}^{\infty} d k_{\|} \frac{1}{2}\left[\delta\left(k_{\|}-k_{0}\right)+\delta\left(k_{\|}+k_{0}\right)\right] \int \frac{d^{2} k_{\perp}}{(2 \pi)^{2}} \\
& \times C\left(k_{\perp}, \tau=0\right) \frac{2 D_{\perp} k_{\perp}^{2}}{D_{\perp}^{2} k_{\perp}^{4}+\left(\omega-k_{\|} U_{\|}\right)^{2}},
\end{aligned}
$$

where $C\left(k_{\perp}, \tau=0\right)$ is the Fourier transform of the exponential decay. Since the plasma equilibrium profiles are not uniform in the perpendicular directions, we introduce the cutoff wave number for the perpendicular diffusion. Then Eq. (83) becomes

$$
\begin{aligned}
S(\omega)= & \frac{1}{2} \int_{k_{c \perp}}^{\infty} d k_{\perp} E\left(k_{\perp}\right)\left[\frac{2 D_{\perp} k_{\perp}^{2}}{D_{\perp}^{2} k_{\perp}^{4}+\left(\omega+\omega_{0}\right)^{2}}\right. \\
& \left.+\frac{2 D_{\perp} k_{\perp}^{2}}{D_{\perp}^{2} k_{\perp}^{4}+\left(\omega-\omega_{0}\right)^{2}}\right],
\end{aligned}
$$

where $\omega_{0}=k_{0} U_{\|}$is the spectrum peak frequency, and the energy spectrum of the exponential decay $E\left(k_{\perp}\right)$ is given by Eq. (61). In view of Eqs. (60) and (65), we see that Eq. (84) gives the shifted autopower spectrum Eq. (72), which is obtained by assuming that there is a dominant frequency $\omega_{0}$ in the autocorrelation function.

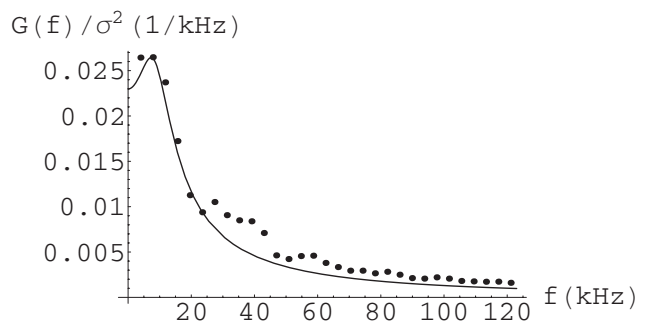

FIG. 6. Comparison of the autopower spectrum. The dots represent the data of the tokamak plasma density fluctuations. The curve is given by Eq. (74). The autopower spectrum $G(f)$ is normalized by the variance $\sigma^{2}$.

\section{COMPARISON WITH EXPERIMENT}

During a steady-state plasma discharge, the fluctuations in the magnetized plasma usually have the following properties $[17,20]$ : the time series of the signals are nearly random, the statistics of the fluctuations are roughly homogeneous and isotropic in the two dimensions perpendicular to the strong magnetic field, and the correlation length is shorter than the length scales of the background density and temperature gradients. Hence we may use Eqs. (70) and (74) to explain the shape and height of the experimental autocorrelations. To compare the theory with experiments, we use the measured variance $\sigma^{2}$ and the observed peak (oscillation) frequency $f_{0}$, and choose the value of the diffusion frequency $f_{d}$, such that the theoretical curves fit the overall shape of the experimental data. Here the fluctuation $\alpha$ is normalized by the equilibrium value, so that the root mean square value $\sqrt{\left\langle\alpha^{2}\right\rangle}$ represents the relative fluctuation amplitude.

As shown in Fig. 6, the theory is compared to the observed plasma density fluctuations in the edge of the Texas experimental tokamak [21]. The time series data were taken from deuterium discharge 230000 at minor radius $27.5 \mathrm{~cm}$, where the limiter was located. From the experimental data, one can obtain the fluctuation amplitude $\sqrt{\left\langle\alpha^{2}\right\rangle} \approx 0.24$ and the peak frequency $f_{0} \approx 8 \mathrm{kHz}$. To fit the experimental data, we choose $f_{d} \approx 5.3 \mathrm{kHz}$ for the diffusion frequency. Thus the quality factor $Q=f_{0} / f_{d} \approx 1.5$, the correlation time $\tau_{c}$ $=1 /\left(6 \pi f_{d}\right) \approx 0.01 \mathrm{~ms}$, and the spectrum width $\Delta f=3 f_{d}$ $\approx 16 \mathrm{kHz}$. The perpendicular correlation length $\lambda_{c \perp}$ near the limiter was about $3 \mathrm{~cm}$ [17]. Thus we estimate the local perpendicular diffusion coefficient $D_{\perp}=f_{d} \lambda_{c \perp}^{2} /(2 \pi)$ $\approx 0.76 \mathrm{~m}^{2} / \mathrm{s}$. Note that the deviations of the experimental data from the theoretical curve probably reflect experimental fluctuations that do not completely satisfy the assumptions of statistical homogeneity and isotropy.

To demonstrate the common features of random fluctuations, we also compare the theory with the observed plasma density fluctuations from helimak experiments [18]. The time series data in Figs. 7 and 8 were taken from argon discharge 508020092 at the radial position $r=110 \mathrm{~cm}$, where the plasma equilibrium profiles have gradual gradients. From the experimental data, one can obtain the fluctuation amplitude $\sqrt{\left\langle\alpha^{2}\right\rangle} \approx 0.23$ and the peak (oscillation) frequency $f_{0}$ $\approx 1410 \mathrm{~Hz}$. To fit the experimental data, we choose $f_{d}$ $\approx 90 \mathrm{~Hz}$ for the diffusion frequency. Thus the quality factor 


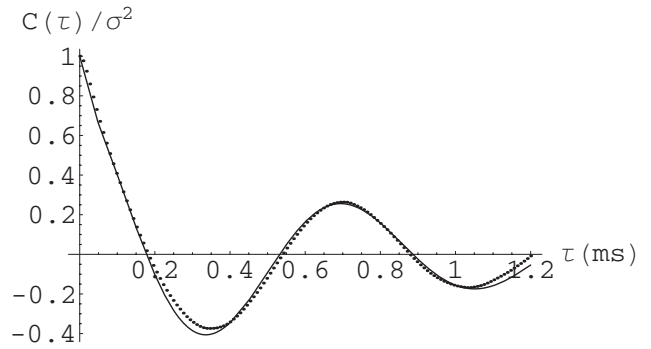

FIG. 7. Comparison of the autocorrelation function. The dots represent the data of the helimak plasma density fluctuations. The curve is given by Eq. (70). The autocorrelation function $C(\tau)$ is normalized by the variance $\sigma^{2}$.

$Q=f_{0} / f_{d} \approx 16$, the correlation time $\tau_{c}=1 /\left(6 \pi f_{d}\right) \approx 0.6 \mathrm{~ms}$, and the spectrum width $\Delta f=3 f_{d} \approx 270 \mathrm{~Hz}$. In the low magnetic field side, the perpendicular correlation length $\lambda_{c \perp}$ $\approx 10 \mathrm{~cm}$. Thus we estimate the local perpendicular diffusion coefficient $D_{\perp}=f_{d} \lambda_{c \perp}^{2} /(2 \pi) \approx 0.14 \mathrm{~m}^{2} / \mathrm{s}$. Note that similar comparison has been done in Ref. [14] for the autocorrelation data at the radial position $r=106 \mathrm{~cm}$.

As shown in Figs. 7 and 8, the temporal correlations of random fluctuations have the following key features. The autocorrelation function has a fast decay in the small $\tau$ region and a slower decay in the large $\tau$ region. Since the power spectrum is the Fourier transform of the correlation function, the corresponding autopower spectrum has a long tail in the high frequency region and a narrow maximum near the peak frequency. Note that one often fits the envelope of the autocorrelation function to a simple exponential decay and approximates the shape of the autopower spectrum by the Lorentzian, i.e., the Fourier transform of an exponential decay. We emphasize that they do not fit the experimental data as well as this noise calculation.

The experiments also observe that the spectral characteristics of plasma density fluctuations depend on the radial positions. As shown in Table I, the correlation times become shorter, in other words, the frequency spectra become wider, when the radial positions move to the edge of the plasma. Presumably the correlation length is constant in this local region. Thus the random walk formula Eq. (69) immediately tells us that the transport coefficients should increase toward the edge of the plasma, as observed in the experiments [1].

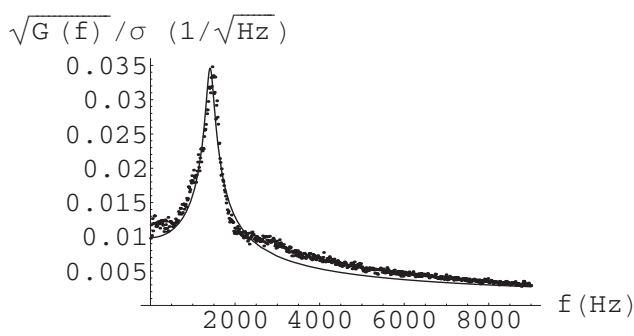

FIG. 8. Comparison of the square root of the autopower spectrum. The dots represent the data of the helimak plasma density fluctuations. The curve is given by the square root of Eq. (74). The autopower spectrum $G(f)$ is normalized by the variance $\sigma^{2}$.
TABLE I. The observed fluctuation amplitude $\sqrt{\left\langle\alpha^{2}\right\rangle}$, the observed peak (oscillation) frequency $f_{0}$, the diffusion frequency $f_{d}$, and the correlation time $\tau_{c}$ of the helimak plasma density fluctuations at different radial positions $r$.

\begin{tabular}{ccccc}
\hline \hline$r(\mathrm{~cm})$ & $\sqrt{\left\langle\alpha^{2}\right\rangle}$ & $f_{0}(\mathrm{~Hz})$ & $f_{d}(\mathrm{~Hz})$ & $\tau_{c}(\mathrm{~ms})$ \\
\hline 114 & 0.33 & 1410 & 90 & 0.6 \\
118 & 0.38 & 1410 & 110 & 0.5 \\
122 & 0.31 & 1410 & 160 & 0.3 \\
\hline \hline
\end{tabular}

\section{SUMMARY AND CONCLUSIONS}

Using noise theory, we have calculated the temporal and spatial correlations of stationary Markovian fluctuations that are statistically homogeneous and isotropic.

In Sec. I, we have reviewed the Wiener-Khintchin theorem and obtained the discrete-index formulas of the power spectra. In Sec. II, we have introduced the following statistical quantities: the correlation function, the power spectrum, the wave-number spectrum, and the wave-number-frequency spectrum. Using the discrete-index formulas, we have derived the general formulas of the wave-number-frequency spectra, which are the essential tools of our theory.

In Sec. III, we have used the diffusion equation to model the Markovian relaxation of fluctuations. For physical systems with finite correlation lengths, the spatial correlations are modeled by the exponential decay. It is found that the energy spectra of the exponential decay are proportional to $k^{-2}$ in the large wave-number range. Assuming that the mean square value of small-scale fluctuations are determined by the wavelengths shorter than the correlation length, we have obtained the analytic results of the autopower spectrum and the autocorrelation function. The results are independent of spatial dimensions. We have found that the diffusion process can give rise to the decay of the correlation function and a broad frequency spectrum of random fluctuations, such as the $1 / f$ noise spectrum. In the diffusion model, the autopower spectrum has a single peak at zero frequency and the autocorrelation function purely decays away. We have also found that the transport coefficients may be estimated by the correlation length and the correlation time. Assuming that there is a dominant frequency in the temporal correlations, the oscillating decay of the autocorrelation function is modeled by a cosine function times the decay due to diffusion. As a consequence, the maximum of the corresponding autopower spectrum is shifted from zero frequency to the dominant frequency.

In Sec. IV, we have used the convection-diffusion equation to describe the relaxation of fluctuations that involves fluid motion and diffusion. We have shown that the product of the wave number and the flow velocity can shift the peak of the spectra away from zero frequency.

In Sec. V, we have investigated the applications of noise theory to plasma fluctuations. The theory has been compared with plasma density fluctuations from the tokamak and helimak experiments. The quantitative comparisons show that the theory can predict the shape of the autocorrelations of the 
observed fluctuations over a broad range of frequencies and time scales. The theory can also relate the amplitude of the frequency spectra to spectral characteristics, such as correlation times. In particular, we have shown that the transport coefficients are proportional to the width of the fluctuation spectra.

\section{ACKNOWLEDGMENTS}

We are grateful to R. D. Bengtson, G. A. Hallock, K. Lee, M. A. Meier, S. M. Mahajan, E. J. Powers, P. E. Phillips, and J. W. Van Dam for helpful discussions. This work was supported by the U.S. Department of Energy.
[1] K. W. Gentle, Rev. Mod. Phys. 67, 809 (1995).

[2] H. B. Callen and T. A. Welton, Phys. Rev. 83, 34 (1951).

[3] L. Onsager and S. Machlup, Phys. Rev. 91, 1505 (1953).

[4] E. C. Taylor and G. G. Comisar, Phys. Rev. 132, 2379 (1963).

[5] P. Dutta and P. M. Horn, Rev. Mod. Phys. 53, 497 (1981).

[6] C. R. Oberman and E. A. Williams, in Basic Plasma Physics, edited by A. A. Galeev and R. N. Sudan (North-Holland, Amsterdam, 1983), Vol. 1, p. 279.

[7] R. H. Kraichnan, Phys. Rev. Lett. 72, 1016 (1994).

[8] W. Horton, Rev. Mod. Phys. 71, 735 (1999).

[9] P. W. Terry, Rev. Mod. Phys. 72, 109 (2000).

[10] J. A. Krommes, Phys. Rep. 360, 1 (2002).

[11] D. Biskamp, Magnetohydrodynamic Turbulence (Cambridge University Press, Cambridge, England, 2003).

[12] M. Lax, Rev. Mod. Phys. 32, 25 (1960).
[13] M. Lax and P. Mengert, J. Phys. Chem. Solids 14, 248 (1960).

[14] B. Li and R. D. Hazeltine, Phys. Rev. E 73, 065402(R) (2006).

[15] R. D. Hazeltine and S. M. Mahajan, Phys. Plasmas 11, 5430 (2004).

[16] R. D. Hazeltine and J. D. Lowrey, Phys. Plasmas 13, 014505 (2006).

[17] A. V. Filippas, R. D. Bengtson, G. X. Li, M. A. Meier, C. P. Ritz, and E. J. Powers, Phys. Plasmas 2, 839 (1995).

[18] J. C. Perez, W. Horton, K. W. Gentle, W. L. Rowan, K. Lee, and R. B. Dahlburg, Phys. Plasmas 13, 032101 (2006).

[19] B. Li, Ph.D. thesis, University of Texas at Austin, 2007.

[20] C. P. Ritz, R. D. Bengtson, S. J. Levinson, and E. J. Powers, Phys. Fluids 27, 2956 (1984).

[21] M. A. Meier, R. D. Bengtson, G. A. Hallock, and A. J. Wootton, Phys. Rev. Lett. 87, 085003 (2001). 